\title{
Cerebral aneurysms: Conflicts and future trends.
}

\author{
Bahaa Ghareeb Hassanin ${ }^{1,}$, Momen M.Almamoun ${ }^{1}$, Mohamed A.Abdalal ${ }^{1}$, \\ Mohamed M. Awesh ${ }^{1}$, Roshdy A.Elkhyat ${ }^{2}$, Alexander Brawanski ${ }^{3}$, Karl- \\ Michael Schebesch ${ }^{3}$. \\ 1 Department of Neurosurgery, Sohag University \\ 2 Department of Neurosurgery, Assuit University \\ 3 Departmenr of Neurosurgery, Regensburg University, Germany
}

\begin{abstract}
Cerebral aneurysms are pouch-like focal dilatations of intracranial arteries, which usually occurred at their branching points. Most of these aneurysms remain asymptomatic and never ruptures. When an aneurysm ruptures, it may bleed into the brain parenchyma resulting in a parenchymal hemorrhage, or more often, it will bleed into the subarachnoid space, resulting in a subarachnoid hemorrhage (SAH). Cerebral aneurysms are not a disease unique to the modern society and a lot of improvements have been achieved especially in its treatment in the second half of the 20th century, especially after the adoption of microsurgical techniques and FDA approval for detachable coils as Endovascular techniques to treat cerebral aneurysms in 1995.

The aim of the current study is to review basic knowledge about the cerebral aneurysms, with brief telescopic view on areas of conflicts, to know the current state of research regarding the cerebral aneurysms to conclude what the future research trends should be.
\end{abstract}

\section{Introduction}

Aneurysms are not a disease unique to modern society. Both ancient literature and bony artifacts dating back to ancient Egypt indicate occurences of aneurysms and at least some extracranial aneurysms were treated. Despite occasional reports, cerebral aneurysms were not wellrecognized as a cause of human illness until the end of the $19^{\text {th }}$ century. ${ }^{(29)}$

Intracranial aneurysms are relatively common. There are four main types of intracranial of $25 \%$ to $50 \%$. Permanent disability occurs in nearly $50 \%$ of the survivors, thus, only approximately onethird of patients who suffer a SAH have a positive outcome. Althought most cerebral aneurysms are asymptomatic and will not rupture, they grow unpredictably and even small aneurysms carry a risk of rupture. (4) There is a great conflict and controversy surrounding the natural history of unruptured aneurysms. It is such a hot topic because knowing the likely course of aneurysms will play a pivotal role in

determining the appropriate management.(24) Treatment options include observation, endovascular coiling, and surgical clipping; microsurgical clipping has been used for the treatment of intracranial aneurysms for longer than 40 years and endovascular coiling devolped a lot in the past 20 years. (38)aneurysms: saccular, fusiform, dissecting, and mycotic type. The saccular type accounts for $97 \%$ of intracranial aneurysms, $85 \%$ of which arises from the arteries of the anterior cerebral circulation. (15)

Symptoms related to unruptured aneurysms mainly occurr due to a mass effect, but the real danger is when an aneurysm ruptures, leading to a subarachnoid haemorrhage, which is a catastrophic medical event with a mortality rate.

Epidemiology of intracranial aneurysms: 
Studies have shown that IAs are common and the overall incidence ranges from $0.4 \%$ to $10 \%$, e.g. in the German population approximately $1.5-$ 2 million people are assumed to harbour a cerebral aneurysms. Frequency increases with age beyond the third decade, incidence is rare in the pediatric population and is pproximately 1.6 times more common in women. The risk of harbouring IAs is 2-4 times higher in families with two or more members with a history of IAs. Most IAs occurences are sporadic and only less than $10 \%$ of patients have a family history of aneurysms. About one-third of all patients with IAs have multiple aneurysms. ${ }^{(29)}$

Although the incidence of IAs is comparable worldwide, their rupture rates are different.The incidence of aneurysmal SAH in most populations is 6-10 cases per 100,000 person/year; in uniated state about 30,000 people suffer a brain aneurysm rupture every year. In other words, there is a brain aneurysm rupturing every 18 minutes.

The rupture rate increases in Finland, Japan and Northern Sweden, the The most plausible pathogenetic theory is that they are acquired due to hemodynamic stress on the relatively unsupported bifurcations of cerebral arteries. This is supported by the clinical observation that many patients with an anterior communicating artery (Acom) aneurysm do have one hypoplastic or absent $\mathrm{Al}$ segment and thus an increased hemodynamic stress on the AcomA occurs. Other factors than hemodynamics and structural alterations of the vessel wall contributing to the development of incidence is $10-16$ cases per 100,000 person/year.This higher incidences may be due to environmental or genetic factors, but reason still remains unknown. ${ }^{(4)}$ Smoking turned out to be a risk factor, but it was not statistically significant. ${ }^{(29)}$

\section{Hypothesis for intracranial aneurysms Formation:}

The majorities of intracranial aneurysms are not considered to be congenital as it has been assumed in the past, but it is generally accepted that IAs are acquired lesions, the actual mechanism of their formation is unknown. ${ }^{(27)}$ The popular theory of a congenital defect in the tunica media of the muscle layer as a weak spot through which the inner layer of the arterial wall would bulge has had doubt cast upon it due to a number of contradictory observations: Gaps in the muscle layer are equally present in patients with and without aneurysms. If an aneurysm has formed, defect in the muscle layer is not located at the neck, but somewhere in the aneurysmal wall of the sac. ${ }^{(27,29)}$

saccular aneurysms may be genetic, infection, vascular trauma, neoplasms, radiation or idiopathic. ${ }^{(20)}$

The role of acquired changes in the arterial wall, in adults, is more likely to happen because there are general risk factors for both subarachnoid hemorrhage (SAH) and development of the aneurysms like hypertension, smoking and alcohol abuse. These factors might contribute to general thickening of the intimal layer in the arterial wall, distal and proximal to branching sites. These "intimal pads" 
are probably the earliest stages of aneurysm formation. Within these pads, the intimal layer is inelastic and therefore causes increased strain of the more elastic portions of the vessel wall. Abnormalities in structural proteins of the extracellular matrix additionally contribute to aneurysm formation. However, it is not known why only some adults develop aneurysms at arterial bifurcations and most do not. ${ }^{(22)}$ Inflammation has been found to have a principal role in the process of IA formation and subsequent rupture. Aspirin with its anti-inflammatory effects was found to be effective in decreasing the risk of aneurysm formation and subsequent $\mathrm{SAH}$ without increasing the risk of intracerebral hemorrhage. Aspirin might be a promising prophylactic drug against IA growth and rupture. ${ }^{(25)}$

Cerebral aneurysms may develop in $10 \%-15 \%$ of patients with polycystic kidney disease, an autosomal dominant disorder. Although Marfan syndrome was previously identified as a risk factor for aneurysms, recent studies do not identify any significant correlation.There are some presumptions on neurofibromatosis type 1 (NFl) and intracranial aneurysms. In a recent study, an association between $\mathrm{NFl}$ and intracranial aneurysms has never been identified in large clinical studies. ${ }^{(5,29)}$

Also, it is suggested that the formation of an intracranial aneurysm is a consequence of a systemic vascular pathology, which is associated with pleomorphism in different candidate genes. Understanding of the mechanisms behind the formation of IAs can provide an opportunity for the development of pharmacological therapy for IAs. ${ }^{(26)}$

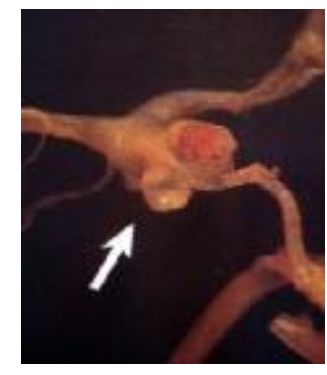

Figure (1). Gross pathology specimen demonstrating saccular aneurysm (arrow) that arises at ICA- PComA Junction (Osborn et al., 1994)

Figure(2). Autopsy of unrecognized basilar bifurcation aneurysms in 2years child, Yasargil 1984

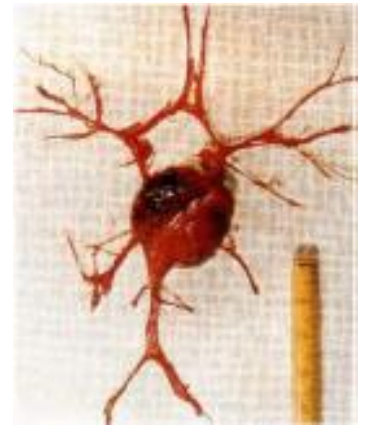

Genetics of intracranial aneurysms:

Many genetic studies have aimed to determine if some genetic variants predispose individuals to the development of IAs. Multinational genome-wide association studies have defined five loci with strong statistical evidence and another 14 loci with suggestive evidence of an association with IAs. 5q26 is one suggestive risk locus for IAs which was also found to be associated with a risk of high systolic blood pressure. Besides, 9p21, which is a general cardiovascular risk locus, was found to be a strong risk locus for IAs. This overlapping genetic background suggests that the formation of IAs might be a part of generalized vasculopathy rather than a separate disease. ${ }^{(11,17)}$ Familial aneurysms are generally larger at time of rupture and more likely to be multiple than sporadic aneurysms. Cerebral aneurysms in patients with a positive family history might result 
from a mesenchymal defect affecting the cerebral vessel wall produced by a lesion of chromosome 16. ${ }^{\text {(37) }}$

\section{Histology of intracranial aneurysms:}

The wall of cerebral arteries is composed of three histologic layers: tunica intima, tunica media and tunica adventitia. The internal elastic lamina (IEL) separates the tunica intima and tunica media. The wall of cerebral arteries differs from that of extracranial arteries in that it lacks the external elastic lamina (EEL) normally present in extracranial arteries between the tunica media and the tunica adventitia. Additionally, the cerebral artries have a paucity of supportive perivascular tissues and fewer smooth muscle cells (SMC) in the tunica media and in IEL than extracranial arteries. Moreover, at the bifurcation of cerebral arteries which is the typical location for the formation of IAs, the tunica media consists mainly of collagen arranged in such a way that it increases resistance to mechanical stress. So the internal elastic lamina is an important layer of the arterial wall in the cerebral vessels.

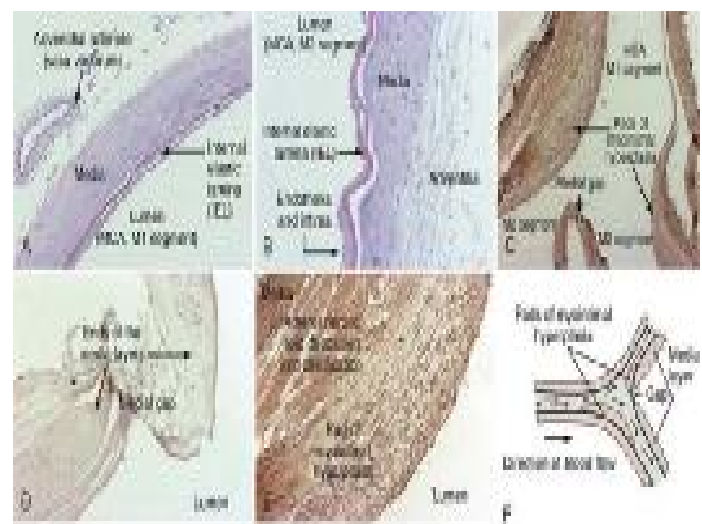

Thus, disruption of this layer due to alterations in hemodynamic and histological characteristics, would promote the formation of aneurysms especially at the regions around the bifurcations. In Helsinki, Frosen et al., four histological wall types for IAs are identified: type A, an endothelialized wall with linearly organized SMC; type $B$, a thickened wall with disorganized SMC; type C, a hypocellular wall; and type D, an extremely thin thrombosislined hypocellular wall. These types probably reflect consecutive stages (A through D) of degeneration suggesting a dynamic nature to the IA wall.

Figure (3).Histology of the intracranial arteries depicting three layers of the intracranial wall ( $A$ and $B$ ) and the presence of medial gaps and pads of myointimal hyperplasia (C-E). $F$, Drawing showing the relative locations of medial gaps and pads of myointimal hyperplasia at a vesselbifurcation.MCA, middle cerebral artery.(From Frosen J. The Pathobiology of Saccular Cerebral Artery Aneurysm Rupture and Repair. Helsinki: University of Helsinki;2006

Rupture of intracranial aneurysms: Most cerebral aneurysms remain clinically silent until they rupture. The International study of unruptured intracranial aneurysms (ISUIA) has been the most comprehensive clinical study of its kind to date, several factors related to the patient and aneurysmspecific characteristics have been examined as possible predisposing factors for the rupture of IAs. In phase II of ISUIA, a higher rupture rate was reported to be associated with larger aneurysms, location in the posterior circulation, and previous history of SAH. ${ }^{(21)}$

\section{Patient-related rupture risk factors:}

Prevalence of aneurysms and rupture risks were shown to increase in both studies due to the factors such as age of the patient; this risk increases accumulatively with age to peak between 40-65 years of age, preexisting familial conditions, in patients 
with a family history of SAH, IAs rupture at a younger age. ${ }^{(3)}$

hypertension is the most frequently reported health condition associated with the formation of IAs and their subsequent rupture and was also found to be a risk factor for multiple IAs and for the rupture of small sized IAs. Smoking has been also associated with higher prevalence aneurysms and its rate of rupture. High alcohol consumption, cocaine use has been found to be significantly associated with aneurysmal SAH. ${ }^{(9,22)}$

IAs are more commonly diagnosed in women than in men. Furthermore, aneurysmal $\mathrm{SAH}$ is more common in women. IAs, in women, carry a higher risk of rupture especially after menopause, which is probably related to the hormonal effects of estrogen on the vascular wall. ${ }^{13,22)}$

Aneurysm-related rupture risk factor:

Although aneurysm size has traditionally been considered a risk factor for the rupture of IAs, a single threshold value for an increased rupture risk has not been defined. Many studies have reported a significantly higher risk of rupture for saccular IAs larger than $10 \mathrm{~mm}$ compared to smaller aneurysms. The ISUIA reports that the rupture rate for aneurysms smaller than $7 \mathrm{~mm}$ was significantly lower than for those larger than $7 \mathrm{~mm}$. However, in clinical experience, it is obvious that many small aneurysms do rupture and about $1 / 3$ of all ruptured aneurysms were smaller than $7 \mathrm{~mm}$ in size. $(21,26)$

Location of the aneurysms has been considered a risk factor for rupture, where posterior circulation aneurysms and aneurysms arising from the posterior communicating artery (PcomA) have a higher risk of rupture relative to anterior circulation aneurysms. Among anterior circulation aneurysms, Acom aneurysms have been reported to have the highest rupture rate, while MCA aneurysms had the lowest rupture rate. ${ }^{(3,33)}$

More recent studies focus on morphological characteristics of aneurysms to predict rupture risks. Several geometric indices, measured by using three-dimensional rotational angiography or digital subtraction angiography correlate with increased rupture rates. (26) The two highest indicators were the aspect ratio (the ratio of the aneurysm's height to its neck) and the volume to neck ratio. ${ }^{(26)}$ other noted two factors include presence of daughter sacs and the non-sphericity index, were strong predictors of aneurysm rupture as well. ${ }^{(26)}$

\section{Mechanism of rupture of intracranial} aneurysms:

It can be concluded that the growth potential of an individual aneurysm is unpredictable and as a result, no aneurysm regardless of size can be considered safe from enlargement and rupture over any period of time. IAs increase in size through real growth rather than stretching which attenuates the wall. Thus, aneurysm size might depict only the degree of wall growth, but not its strength. Wall morphology, on the other hand, correlates more strongly with the quality of wall growth and its resistance to rupture. ${ }^{(27)}$

It is generally accepted that rupture occurs when the intra-aneurysmal hemodynamic stress surpasses its wall resistance. ${ }^{(27)}$ other factors, such as complement activation and protein kinases are involved in IA growth and rupture. Although several hypotheses have been postulated to explain this association, the underlying mechanism 
is still unproven. Histological, genetic, immunological and molecular biologic studies are still needed to explain the relationship between the morphological characteristics of IAs and their rupture. Besides, large-scale clinical studies are needed to evaluate the predictive value of other morphological variables related to the risk of rupture. $(18,31)$

\section{Aneurysms Associated with} Arteriovenous Malformations:

There is an increased incidence, or better, an increased amount of visible aneurysms associated with arteriovenous malformations. The incidence of these aneurysms in AVMs is up to $25 \%$. Approximately $50 \%$ of these aneurysms are located on a feeding artery, $25 \%$ within the nidus. ${ }^{(28)}$ Flow related aneurysms probably develop due to hemodynamic stress caused by increased flow and pressure, with subsequent dilatation and pathologic changes in feeding arteries. AVM-associated aneurysms contribute to an increased risk of hemorrhage. A $7 \%$ risk of hemorrhage for these combined lesions is estimated compared to a $1.7 \%-3 \%$ risk for AVMs without associated aneurysms. ${ }^{(28)}$

In case of rupture, the hemorrhage is more often located intraparenchymally than subarachnoidally. Management of these combined lesions is still debatable. In fact, hemodynamics change after elimination of the AVM might place the aneurysm at risk. On the other hand, proximal asymptomatic aneurysms may regress after removal of the AVM. However, aneurysms located in the posterior circulation associated with an AVM are at higher risk of rupture and therefore should be treated as soon as possible even if they had not ruptured before. ${ }^{(28,35)}$
Figure (4) aneurysms associated AVM, sites in relation to AVM, Nakahara I 1999

\section{Rebleeding after SAH:}

Rebleeding is a frequent and sometimes devastating neurologic complication of SAH which clinically coexist with new

A

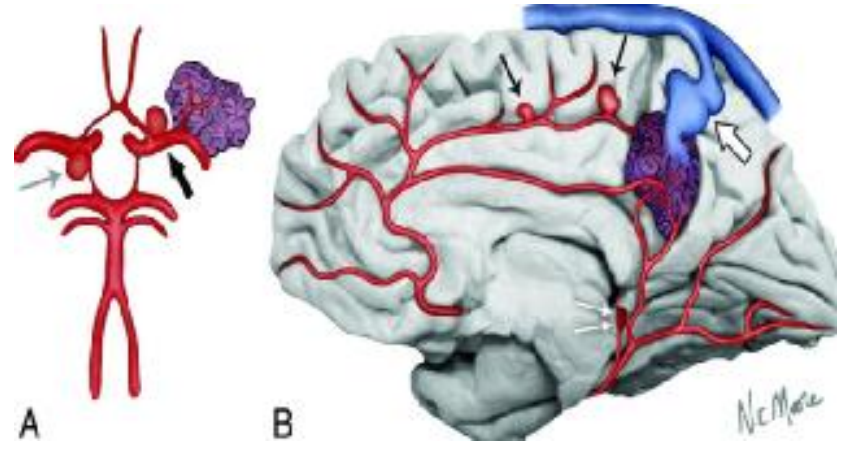

neurologic deficits. It is postulated to be due to breakdown of perianeurysmal clots as clot formation and tissue damage stimulate fibrinolytic activity in the CSF. The efficacy of antifibrinolytic therapy such as aminocaproic acid and tranexamic acid to prevent rebleeding showed a significantly decreased incidence of rebleeding, however, mortality was not altered, and this therapeutic approach was associated with an increased risk of delayed cerebral ischemia, embolism, and deep venous thrombosis. ${ }^{(3,33)}$

Since early rebleedings commonly occur before the first CT scan is obtained, the true frequency of early rebleeding is definitely underestimated. As many as $20 \%$ of patients may rebleed within the first 2 weeks, one third in the first month, and 50\% will rebleed within 6 months, if the aneurysm is not treated and the mo)

Women have a 2.2 times higher recurrence rate of hemorrhage than men.The worse the clinical grade on admission, the more likely rebleeding occurs. Hypertension increased the 
likelihood of rebleeding among patients. Of patients with a diastolic blood pressure below $90 \mathrm{~mm} \mathrm{Hg}, 25 \%$ rebleed, whereas of those with a diastolic pressure above 109, 75\% rebleed. Older patients are more prone to rebleeding. Aneurysms pointing down are less likely to rebleed than those pointing up in the direction of the jet stream of the blood. Short broad aneurysms rebleed more frequently than long narrow aneurysms. Posterior communicating artery aneurysms rebleed at a higher rate than do anterior communicating artery and vertebrobasilar aneurysms. ${ }^{(30)}$

Vasospasm related to SAH:

Vasospasm is a major cause of morbidity and mortality in patients after $\mathrm{SAH}$ and is often associated with delayed cerebral ischemia. However, many patients are asymptomatic despite various degrees of angiographically visible vasospasms. Although vasospasm is noted angiographically in $70 \%$ of patients after $\mathrm{SAH}$, it becomes symptomatic only in about half of those patients. This difference probably suggests additional factors determining whether and where secondary cerebral ischemia occurs like the degree of arterial narrowing, its location and the adequacy of collateral blood flow, as well as other factors such as increased intracranial pressure or the presence of cerebral oedema. ${ }^{(8)}$

Vasospasm is best detected on angiograms. However, transcranial Doppler ultrasound is the method of choice to monitor blood flow velocities in patients after SAH. CTA and MRA role has not been determined in this subgroup of patients. A CT perfusion is feasible in detecting vasospasm and might even predict outcome. ${ }^{(8,19)}$
Despite intensive research, the pathogenesis of vasospasm has not been entirely elucidated. Releases of yet unidentified factors into the subarachnoid space are considered to induce vasospasm and subsequent cerebral ischemia. There is a widespread postulation of a close relationship between the amount of subarachnoid blood clots and the degree of vasospasm and delayed cerebral ischemia. However, there are several arguments against these assumptions; subarachnoid blood is not a predictor of vasospasm perse, since vasospasm and delayed cerebral ischemia rarely occur in patients with SAH after rupture of an AVM or perimesencephalic SAH, one would expect a lower incidence of vasospasm after clipping compared to coiling, since there is no way to remove a subarachnoidal clot during coiling. But this effect has not been observed. Furthermore, the site of delayed cerebral ischemia does not always correspond with the distribution of subarachnoid blood. ${ }^{(8,14)}$

\section{Unruptured cerebral Aneurysms:}

Asymptomatic aneurysms may be defined as additional aneurysms found in patients with another symptomatic aneurysm, which are not responsible for the clinical symptoms or those aneurysms found in patients investigated because they are at risk of harbouring an aneurysm and those found unexpectedly in patients undergoing investigation for any other suspected pathology or unrelated clinical symptoms. ${ }^{(1)}$

Depending on the location of an unruptured aneurysm it can be completely asymptomatic. On the other hand, unruptured aneurysms can cause neurologic symptoms while touching or transmitting pulsation to cranial nerves 
or other cerebral structures. Symptoms can be pain, cranial nerve palsies, visual disturbances, dysesthesia, vertigo and seizures. Thromboembolism ischemic events can occur distal to both small and large unruptured intracranial aneurysms (predominantly in the anterior circulation) were observed in $3.3 \%{ }^{(16)}$

Symptomatic unruptured aneurysmsare usually larger than incidental aneurysms and are often discovered near to the skull base where they are more likely to affect cranial nerves. The most frequent affected cranial nerves are the ocuoculomotor nerve and the optic nerve. ${ }^{(24)}$

Given the high mortality and morbidity associated with an aneurysm rupture, it is crucial to determine the likelihood of rupture to decide whether to treat an aneurysm or not. The findings of the ISUA have a lot of criticism and failed to solve these issues and redefine the critical aneurysm size from $10 \mathrm{~mm}$ down to $7 \mathrm{~mm}$. ${ }^{(35)}$
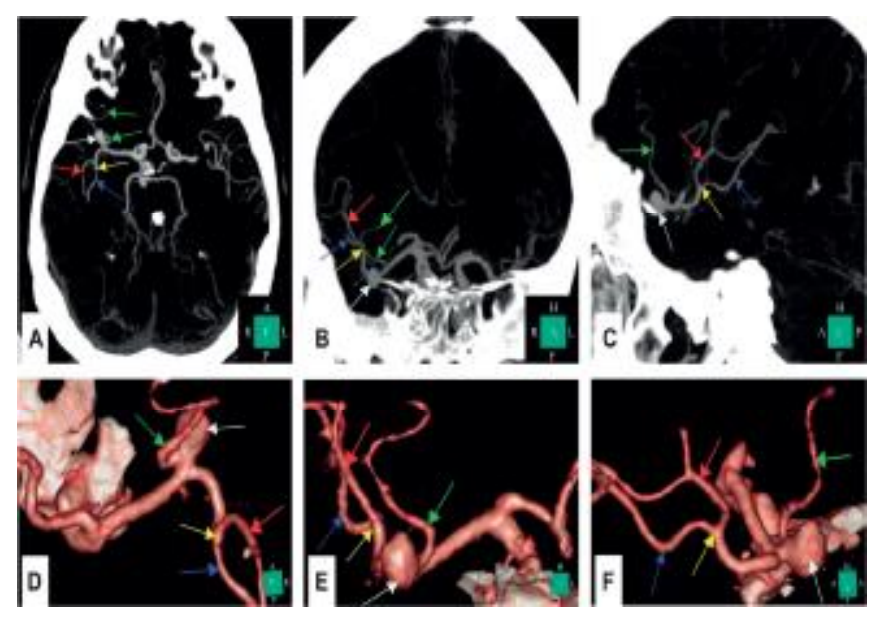

cortical branch (green arrow) just proximal to the right MCA. Hernisnemi 2011.

Transcranial Ultrasound in cerebral aneurysms:

Although the technique is quick, safe, inexpensive and non-invasive, it is highly dependent on the skills of the operator. At the moment, TCD for the detection of cerebral aneurysms is only of scientific interest and cannot be recommended for routine use. In fact, it does not play any role in diagnostic work-up of SAH patients or in screening. (12) TCD is used to study changes in cerebral arterial flow velocity after subarachnoid hemorrhage and after surgery of cerebral aneurysms. A steep early increase in flow velocity seems to predict future ischemia. Velocities over $200 \mathrm{~cm}$ per second almost always occur in patients with severe vasospasm. Flow velocitie is only a rough guide to vasospasm and must take in account the clinical conditions and available radiological imaging. ${ }^{(34)}$

Electroencephalography (EEG) in cerebral aneurysms:

While EEG has little use in the diagnosis and localization of ruptured cerebral aneurysm, it nevertheless finds some application in the evaluation of brain function in patients who are obtunded or comatose following subarachnoid hemorrhage. ${ }^{(36)}$

Van der Drift (1961) summarized the findings on EEG in patients with ruptured cerebral aneurysm and noted that EEG findings basically paralleled the disturbances in cerebral blood flow, but noted that the EEG tracing tended to normalize within 6 weeks of hemorrhage while blood flow alterations could persist. ${ }^{(32)}$

In an interesting recent case, in the author's series, a 45-year-old woman presented with subarachnoid 
hemorrhage and angiography revealed bilateral MCA aneurysms. helpful in determining which of the lesions had bled. An EEG, however, showed dysfunction on the left cerebral hemisphere. Because the right-sided aneurysm was significantly larger than the left, operation was carried out on the right side and an unruptured aneurysm was found. Subsequent left sided exploration revealed the aneurysm which had bled. In this case EEG proved more helpful than angiography for localization of hemorrhage. Techniques such as this might find application in the prognosis of comatosed patients and in determining case selection for operation. ${ }^{(32)}$

\section{Positron Emission Tomography in cerebral aneurysms:}

Positron Emission Tomography or PET scanning is an in vivo autoradiographic technique for measuring cerebral blood flow and metabolism. A variety of radioactive short half-life materials are injected intra arterial necessitating the immediate availability of a cyclotron. This drastically limits the widespread use of PET scanning, currently confined to research purposes. With its ability to quantitate cerebral blood flow and metabolism, PET scanning is being applied to aneurysm patients with associated vasospasm. Efforts are underway to demonstrate that symptomatic patients with vasospasm have an uncoupling of cerebral blood flow and metabolism. Future work with this new investigative device will certainly shed light on the pathophysiology of subarachnoid hemorrhage induced vasospasm. ${ }^{(31,33)}$

\section{Management of aneurysmal SAH:}

In addition to the prevention of rebleeding, the main goal of treatment for patients with ruptured IAs is to
Computerized tomography was not

manage the complications associated with SAH, in particular, vasospasm. For vasospasm prophylaxis, oral nimodipine is traditionally administered and prescribed to patients for three weeks once aneurysmal SAH is diagnosed. Nimpodipine was associated with a $40 \%$ reduction in poor outcomes after an aneurysmal SAH. Other drugs, such as magnesium sulphate and statins, are occasionally used, but with fewer reported benefits. The use of triple- $\mathrm{H}$ therapy (hypervolemia, hemodilution and hypertension) is more controversial and is usually reserved for symptomatic patients. Endovascular mechanical and/or pharmacological angioplasty is increasingly being used to treat cerebral vasospasms. ${ }^{(6)}$

Hypertension, which may be the precipitating event that leads to the rupture of the aneurysm, is often a longstanding issue for patients with SAH. Thus, blood pressure control may be relative to a patient's baseline blood pressure. To avoid lowering blood pressure excessively, cerebral perfusion pressure (CPP) must be maintained at adequate levels. The American Heart Association (AHA) 2012 Guidelines for the Management of Aneurysmal SAH do not give any specific blood pressure recommendation. However, general recommendations call for systolic blood pressure $<160 \mathrm{mmHg}$ because of the risk of rebleeding. ${ }^{(7)}$

Nimodipine is recommended by AHA for all patients with SAH for vasospasm prophylaxis. Although it has not been shown to improve cerebral vasospasm by angiogram, nimodipine has decreased delayed ischemia and improved neurologic outcomes. Verapamil has been shown to improve neurologic 
outcomes without increasing ICP. Erythropoietin has shown some promise in lowering the incidence of vasospasm and DCI, it also has shown improved outcomes, but further studies are needed to confirm this result. ${ }^{(6,7)}$

Up to $26 \%$ of patients with SAH experience seizure-like episodes. Some small, nonrandomized studies have shown that prophylactic use of anticonvulsant therapy in the immediate posthemorrhage period may be beneficial,

but definitive research suggests its efficacy is lacking. If such therapy is used, only a short course (3-7 days) is recommended. ${ }^{(7)}$

Anemia is common in SAH patients. The average drop in hemoglobin concentration in SAH patients is $3 \mathrm{~g} / \mathrm{dL}$. Higher hemoglobin levels have been associated with positive functional outcome. Blood transfusions, however, carry associated risks, such as an impaired immune system and increased incidence of infection. The Neurocritical Care Society recommends maintaining hemoglobin between $8-10 \mathrm{~g} / \mathrm{dL}$ and maintaining higher levels (up to 12 $\mathrm{g} / \mathrm{dL}$ ) for patients at risk for DCI. ${ }^{(6)}$

When aneurysm obliteration is delayed, antifibrinolytic drugs such as aminocaproic acid or tranexamic acid have been shown to reduce the incidence of rebleeding, although neither of these drugs is approved by the United States FDA for this use. ${ }^{(6,7)}$ Data suggest that treatment with statins may help reduce the size and growth progression of cerebral aneurysms, but more investigation is necessary to determine dosing, as conflicting data show that statins may increase aneurysm size at higher doses. ${ }^{(7)}$

Time of Aneurysm Treatment after Subarachnoid Hemorrhage:
Over many years, the most suitable timing of ruptured aneurysm surgery has been debatable. In the 1960s and 1970s, most patients were operated on in the late phase 2 or 3 weeks after the hemorrhage as surgery in the initial 2 weeks was considered too dangerous, because operating on swollen and vulnerable brain tissues led to high rates of perioperative complications. This was particularly true during the period of maximum vasospasm between days 4 and 10 which was considered a particularly dangerous period to operate, because of the risk of delayed cerebral ischemia (DCI). Extensive evidence is available demonstrating that early surgery within 72 hours after the onset of SAH is associated with improved outcome among patients with ruptured cerebral aneurysms in the anterior circulation. The ideal timing of surgery for cerebral aneurysms in the posterior circulation is still controversial. A Cohrane review in 2001 identifies only 1 randomized clinical trial on timing of surgery after aneurysmal SAH. In this trial, patients undergoing early surgeries (days 0-3) tended to have the best outcome, and patients undergoing intermediate surgeries (days 4-7) the worst. A systematic review that included not only this trial, but also 10 observational studies in 2012, concludes that both early (days 0-3) and intermediate (days 4-7) surgical treatments resulted in better outcomes than did late surgery. In conclusion, it is evident that aneurysm treatment after day 10 is associated with worse outcome, regardless of treatment modality and advocates treatment of the (39)

Interestingly, microsurgical clipping between days 5 to 10 did not lead to a 
higher chance of DCI, whereas coiling between days 5 to 10 did increase the chance of DCI, but not poor outcome. Based on these results, it is not recommend to postpone clipping until day 10 or later in patients who are candidates for aneurysm treatment earlier. ${ }^{(39)}$

\section{Treatment of Unruptured cerebral Aneurysms:}

This is still a controversial topic and no solid indications up till now have been in agreement about. The management of asymptomatic unruptured aneurysms remains controversial and depends on a full understanding of their natural history balanced against the risks of treatment and long-term protection afforded. ${ }^{(15)}$ unfortunately, unruptured aneurysms are a heterogeneous entity, both in terms of morphology and behaviour, e.g. tendency to rupture. Aneurysm size, certain locations with an increased risk of rupture perse: posterior circulation aneurysms and those aneurysms arising from the pcomm, hypertension, aneurysm multiplicity, multilobular aneurysm, female sex, heavy alcohol consumption, smoking, and ruptured aneurysm in another location being specific risk factors for a higher probability of rupture and therefore treatment is indicated. ${ }^{(24)}$

Surgical and endovascular treatment of unruptured aneurysms demonstrated that the costs treating an unruptured aneurysm are significantly lower than treating patients with SAH regarding length of hospital stay and sequelae of morbidity and mortality. ${ }^{(15,35)}$

Currently, healthcare is undergoing a major reorganization to meet growing economic pressure and the aspect of preventive therapy becomes more and more important. Therefore, indication for treatment of an unruptured aneurysm has to be considered in several respects: what is the risk of aneurysm rupture and what are the costs to treat a subarachnoid hemorrhage? What are the costs of treating an unruptured aneurysm either neurosurgically or via an endovascular approach to avoid SAH with possibly fatal complications? Rising costs of treating an aneurysmal hemorrhage have to be weighted against the risk of rupture of an incidentally detected aneurysm ${ }^{(15)}$

\section{Surgical vs Endovascular management outcome of treatment:}

For some aneurysms, endovascular treatment may be preferred, whereas for others microsurgery is still superior in both cost and outcomes. The choice of open surgery vs. endovascular techniques to treat cerebral aneurysms, remains an individualized decision. Many factors play a role in treatment decision making: age, past medical history, and medical/neurological conditions help to determine the patient's ability to tolerate a specific treatment. For example, an old patient with a ruptured aneurysm in poor neurological and general condition, coil embolization may be superior to surgery in this setting. On the other hand, a young patient with an unruptured aneurysm may be a good candidate for open surgery in anticipation of achieving a durable result. Aneurysm characteristics such as location (suitability for surgical access), morphology, and size i.e MCA aneurysms tend to have complex morphologies with wide necks and important arterial branches may be incorporated into the aneurysm, these aneurysms tend to be more suitable for open surgery. Also, a ruptured aneurysm with a large intracerebral hematoma and mass effect may be better suited for 
surgery. Patient preference also plays a role in which treatment is chosen in some instances. Operator preferences are often understated but equally important, this includes the operator's bias toward a certain treatment because of experience and familiarity. Some complex aneurysms may require a combination of open and endovascular surgeries. ${ }^{(23)}$

Meta-analysis of multicenter randomized studies includes ISAT that compares the outcomes of ruptured cerebral aneurysms treated either via microsurgical clipping or by endovascular therapy, illustrates that the Initial clinical outcomes indicate little differences between endovascular and surgical treatment for aneurysms of the anterior circulation but that coiling results yield better outcomes for those of the posterior circulation. The effect on mortality rates is not statistically different across the two treatments. Rebleeding rates within the first month were higher in patients allocated to endovascular coil embolization. ${ }^{(21)}$

Regarding the occlusion of ruptured aneurysms, microsurgical clipping procedure is significantly superior. Initial subtotal obliteration is stated in $1.6 \%$ of the microsurgical cases versus $19.1 \%$ of the endovascular cases. In ISAT, the complete occlusion rate in the endovascular group (discharge until over 2 years) was $66 \%$ of the treated aneurysms. Retreatment was performed on $17.4 \%$ of the patients who underwent endovascular therapy and on $3.8 \%$ of the patients who underwent surgical clipping. Long term follow ups indicated that endovascular coiling had a higher risk of rebleeding than clipping $(6.1 \%$ versus $0.4 \%$ ), but the risk was low and remained similar to the risk of $\mathrm{SAH}$ from another aneurysm. ${ }^{(21,38)}$
For unruptured cerebral aneurysms, endovascular coiling and surgical clipping have similar risk ratios of mortality, bleeding, cerebral ischemia, independence in daily activities, and further investigation is needed on quality of life and cognitive outcome. Despite the major advances in endovascular techniques, it still bears a lower rate of occlusion and microsurgical clipping is still the most efficient procedure. ${ }^{(21)}$

Long term rupture rates for unruptured aneurysms in patients without a history of $\mathrm{SAH}$ and aneurysms located in the internal carotid artery, Acom or ACA, or MCA were $0 \%, 2.6 \%, 14.5 \%$, and $40 \%$ for aneurysms less than $7 \mathrm{~mm}, 7-12$ $\mathrm{mm}, 13-24 \mathrm{~mm}$, and $25 \mathrm{~mm}$ or greater, respectively, compared with rates of $2.5 \%, 14.5 \%, 18.4 \%$, and $50 \%$, respectively, for the same-size categories involving posterior circulation and Pcom aneurysms. These rates often equal or exceed the risks associated with surgical or endovascular repair of comparable lesions. Patients' age is a strong predictor of surgical outcomes, and the size and location of an aneurysm predict both surgical and endovascular outcomes. ${ }^{(24)}$

Van Rooij et al noticed that, the significantly lower absolute rate of symptomatic ischemic stroke is (endovascular treatment $19.7 \%$ versus microsurgical clipping 5.3\%). Also, the safety and efficacy profile of flow diversion should discourage the use of these devices in aneurysms that can be treated with other techniques. Direct mortality was not significantly different between the two groups (endovascular group: $0.9 \%$ microsurgical clipping group: $0.7 \%){ }^{(15,35)}$

Although the ISAT study indicated that endovascular treatment was a safe and effective alternative to surgical treatment 
for certain ruptured aneurysms, the results were not sufficient to determine that endovascular treatment is superior to surgical treatment in all patients with ruptured aneurysms and cannot be applied to unruptured aneurysms. ${ }^{(35)}$

Screening for Intracranial Aneurysms: Of the whole population, about $2 \%$ harbor cerebral aneurysms. In most of cases, the aneurysm is not prone to rupture and usually remains asymptomatic throughout their life. Given the serious consequences of intracranial aneurysmal rupture with catastrophic consequences, with up to a $50 \%$ mortality rate even with the best available management and the emergence of new technologies that can aid diagnosis and treatment before rupture, more attention to screening unruptured cerebral aneurysms is mandatory. ${ }^{(2)}$

Screening asymptomatic without risk factors or even those with acquired risk factors, such as smoking or alcohol abuse do not seem to provide any benefits. ${ }^{(10)}$ Screening the entire population for IAs is not logical, since most of them do not harbors IAs. Furthermore, treating all diagnosed IAs is unnecessary since most of them will never harm the patients, parellal to the cost, time, and potential hazards of screening tools. On the contrary, waiting till IAs rupture is not justifiable, as that leaves most patients dead or disabled. ${ }^{(2,}$ 35)

Screening IAs in patients with positive family history of ruptured intracranial aneurysm is controversial. For patients with one affected first-degree relative, the Stroke Council of the American Heart Association does not recommend screening aneurysms. ${ }^{(4)}$ Screening patients with positive two or more family members with intracranial aneurysms is more complexed. Several studies ${ }^{(16)}$ advocate screening for such patient population, based on the higher rupture rates. But more recent studies indicate that screening does not have a significant reduction of the morbidity or mortality rates for those patients. Therefore, the decision of whether or not to screen is best decided on a case-bycase basis. $^{(2)}$

This conflict leads to a major clinical dilemma that should be resolved in future research when more is learned about the risk of IAs rupture and the techniques for diagnosis and management of intracranial aneurysms are improved. Screening should also be considered for patients with rare conditions (e.g. autosomal dominant polycystic kidney disease) that are associated with an increased risk of aneurysms. However, it should be based on their overall health. In patients with a history of aneurysmal subarachnoid hemorrhage, the annual rate of new aneurysm formation is between 1 and 2 percent, and the risk of aneurysmal rupture appears to be increased. (37) Therefore, surveillance of these patients with magnetic resonance angiography or intra-arterial digital subtraction angiography may be justified. ${ }^{(2,4)}$

\section{Summar and trends for the future:}

From the overview on the review of the literature, it can be concluded that:

- In the past decade a lot of progress has been achieved to improve the modalities and techniques to treat cerebral ameurysms; microsurgical clipping become more efficient, less invassive and direct toward eliminating cerebral aneurysms with minimal trauma to the brain, normal vessles and surrounding tissues through improved orientation and understanding the brain 
microanatomy, advance in microscope and microinstruments parellal to improved handling under high power of magnification through tiny gabs, at the same time progress in endovascular techniques become more complexed from just coil packing to use of ballon or stent and flow diverters with the thromboembolic risk and still carry a low aneurysm occlusion rate.

- The exact mechanism for cerebral aneurysms formation is still unclear, the role of inflammatory and genetics process should be extensively examined. So, it might be possible to develop pharmaceutical therapies that would reverse the process of IA formation

- When aneurysm is formed, some aneurysms rupture while others do not. Many studies have focused on the morphological characteristics of aneurysms on 3D angiography to predict rupture risks. Other researches correlate some morphological characteristics with the histology of the aneurysms wall in order to determine more reliable indicators for the degree of resilience of the aneurysm wall to rupture. All available data, however, is suggestive rather than conclusive. Integration of both, meticulous examination of the aneurysm tissue provided during microsurgical clipping by histochemical and immunological studies and the newly developed investigation tools such as the ultrahigh-field MRI, that would probably enable examination of the aneurysm wall for weak spots, would allow for more reliability when classifying IAs as either stable or rupture-risk harboring.

- Prevention of rebleeding after SAH is a big challang. Also, the efficacy of antifibrinolytic therapy such as aminocaproic acid and tranexamic acid to prevent rebleeding and the benefit of statin to control the growth of the aneurysm is still conflicting.

- Issues like the pathogenesis of vasospasm due to $\mathrm{SAH}$ and why vasospasm and delayed cerebral ischemia occure in rupture aneurysm SAH and rarely occur in patients with SAH after rupture of an AVM or perimesencephalic $\mathrm{SAH}$, have not been entirely elucidated.

- Screening for cerebral aneurysm represents a clinical dilama, as only about $2 \%$ of the whole population are expected to harbor IAs. In most cases, however, it will not rupture or harm the patient. Also, it is an expensive and time-consuming procedure that bears the hazards of radiation exposure. Despit that, waiting till an aneurysm rupture occurs is not justifiable, as it consequences can be catastrophic. Indentifying the genetic factors associated with IAs could make screening for them as fast and as smooth as a simple blood test, resulting in a higher percentage of patients with IAs being identified early. Untill that happens, a low threshold to investigate patients with any CNS symptoms should be adopted.

- Preventive medicine is the best route. Despit that, there is no solid indication to treat unrupture cerebral aneurysm due to missing real rupture risk.

- All scores are routinely used to evaluate outcomes after cerebral aneurysms treatments, and only measure recovery after any brain insult, irrespective to the efficacy of 
the treatment modality in eliminating the absolute and relative risk of aneurysm ruptures. So an urgent need arises for high quality evidence and stratification scale to clearly evaluate the safety and efficacy of these techniques, and to determine which treatment provides the best quality and outcomes for patients.

- Advances in endovascular technology might provide for the development of new polymer-coated stents and flow diverters which do not require cotreatment with antiplatelet drugs. Furthermore, it is not impossible that some of today's science fiction might come true resulting in scientific breakthroughs in the future, whereby we witness nanorobots navigating throughout our cerebral arteries to treat IAs or strengthen their walls

\section{Referances:}

A;

1- Ajay Malhotra, MD, MMM *; Xiao Wu, $B S$ *; Howard P. Forman, MD, MBA; Holly $K . \quad$ Grossetta Nardini, MLS; Charles C. Matouk, MD; Dheeraj Gandhi, MD; Christopher Moore, MD; Pina Sanelli, MD, MPH Ann Intern Med; Growth and Rupture Risk of Small Unruptured Intracranial Aneurysms: A Systematic Review; 2017;167(1):2633.DOI: 10.7326/M17-0246

Published at www.annals.org on 6 June 2017@2017 American college of physicians.

B;

2- Bossuyt PM, Raaymakers TW, Bonsel GJ, Rinkel GJ (2005) Screening families for intracranial aneurysms: anxiety, perceived risk, and informed choice. Prev Med 41(3/4):795-799.

3- Broderick, J.P., C.M. Viscoli, T. Brott, W.N. Kernan, L.M. Brass, E. Feldmann, L.B. Morgenstern, J.L. Wilterdink, R.I. Horwitz, and I. Hemorrhagic Stroke Project, Major risk factorsfor aneurysmal subarachnoid hemorrhage in the young are modifiable. Stroke,2003 34(6): p. 1375-81.

C ;

4- Charles Vega,M.D, Jeremiah V Kwoon, M.D and Sean D. Lavine, M.D. University of California, Irvine, College of Medicine, Irvine, California; Intracranial Aneurysms: Current Evidence and Clinical Practice; Am Fam Physician. 2002 Aug 15;66(4):601-609.

5- Conway JE, Hutchins GM, Tamargo RJ (2001) Lack of evidence for an association between neurofi bromatosis typeI and intracranial aneurysms: autopsy study and review of the literature. Stroke 32:2481-2485.

D ;

6- Dankbaar, J.W., A.J. Slooter, G.J. Rinkel, and I.C. Schaaf, Effect of different componentsof triple-H therapy on cerebral perfusion inpatients with aneurysmal subarachnoid haemorrhage:a systematic review. Crit Care, 2010. 14(1): p. R23.

7- Diringer MN, Bleck TP. Claude Hemphill $\mathrm{J} 3^{\text {rd }}$, et al. Critical care management of patients following aneurysmal subarachnoid hemorrhage: recommendations from the Neurocritical Care Society's Multidisciplinary Consensus Conference. Neurocrit Care. 2011 Sep;15(2):211-240.

E ;

8- Eddleman, C.S., M.C. Hurley, A.M. Naidech, H.H. Batjer, and B.R. Bendok, Endovascularoptions in the treatment of delayed ischemic neurological deficits due to cerebral vasospasm. Neurosurg Focus, 2009. 26(3): p.E6.

F ;

9- Fogelholm, R., J. Hernesniemi, and M. Vapalahti, Impact of early surgery on outcome after aneurysmal subarachnoid hemorrhage. A population-based study.Stroke, 1993. 24(11): p.1649-54.

10- Frosen, J., A. Piippo, A. Paetau, M. Kangasniemi, M. Niemela, J. Hernesniemi, and J. Jaaskelainen, Remodeling of saccular cerebralartery aneurysm wall is associated with rupture: histological analysis of 24 unruptured 
and 42ruptured cases. Stroke, 2004. 35(10): p. 2287-93.

G;

11- Gaal, E.I., On the genetics of intracranial aneurysms and on growth factor induced angiogenesis in the murine brain, in Neurosurgery.2012, Helsinki university Helsinki.

12- Griewing B, Motsch L, Piek J, Schminke U, Brassel F, Kessler C (1998) Transcranial power mode Doppler duplex sonography of intracranial aneurysms. J Neuroimaging 8:155-158

$\mathrm{H}$;

13- Harrod, C.G., H.H. Batjer, and B.R. Bendok, Deficiencies in estrogenmediated regulation of cerebrovascular homeostasis may contribute to an increased risk of cerebral aneurysm pathogenesis and rupture in menopausal and postmenopausal women. Med Hypotheses, 2006. 66(4): p. 736-56.

14- Hoh BL, Topcuoglu MA, Singhal AB, Pryor JC, Rabinov JD, Rordorf GA, Carter BS, Ogilvy CS (2004) Effect of clipping, craniotomy, or intravascular coiling on cerebral vasospasm and patient outcome after aneurysmal subarachnoid hemorrhage. Neurosurgery 55(4):779-786; discussion 786-789

$\mathrm{J}$

15- J.B. Bederson, I.A. Awad, D.O. Wiebers, D. Piepgras, E.C. Haley Jr., T. Brott, et al. Recommendations for the management of patients with unruptured intracranial aneurysms: a statement for health care professionals from the Stroke Council of the American Heart AssociationCirculation, 102 (2000), p. 2300

16- Juvela S., Poussa K., Porras M. (2001). Factors affecting formation and growth of intracranial aneurysms: a longterm follow-up study. Stroke 32, 48549110.1161/01.STR.32.8.1933

K;

17- Korja, M., K. Silventoinen, P. McCarron, S. Zdravkovic, A. Skytthe, A. Haapanen, U.de Faire, N.L. Pedersen, K. Christensen, M. Koskenvuo, J. Kaprio, and E.P. Genom, Geneticepidemiology of

spontaneous

subarachnoidhemorrhage: Nordic Twin Study. Stroke, 2010. 41(11): p. 2458-62.

L;

18- Laaksamo, E., R. Tulamo, M. Baumann, R. Dashti, J. Hernesniemi, S. Juvela, M. Niemela, and A.Laakso, Involvement of mitogen-activated protein kinase signaling ingrowth and rupture of human intracranial aneurysms.Stroke, 2008. 39(3): p. 886-92.

19- Laslo AM, Eastwood JD, Chen FX, Lee TY (2006) Dynamic CT perfusion imaging in subarachnoid hemorrhagerelated vasospasm. AJNR Am J Neuroradiol 27(3):624-631

M;

20- Meng H., Wang Z., Hoi Y., Gao L., Metaxa E., Swartz D. D., Kolega J. (2007). Complex hemodynamics at the apex of an arterial bifurcation induces vascular remodeling resembling cerebral aneurysm initiation. Stroke 38, 1924 193110.1161/STROKEAHA.106.481234

21- Molyneux A, Kerr R, Stratton I, et al. International Subarachnoid Aneurysm Trial (ISAT) Collaborative Group. International Subarachnoid Aneurysm Trial (ISAT) of neurosurgical clipping versus endovascular coiling in 2143 patients with ruptured intracranial aneurysms: a randomised trial. Lancet. 2002;360:1267-74.

22- Morita, A., S. Fujiwara, K. Hashi, H. Ohtsu, and T. Kirino, Risk of rupture associated with intact cerebral aneurysms in the Japanesepopulation: a systematic review of the literature from Japan. J Neurosurg, 2005. 102(4): p. 601- 6.

Q;

23- Qureshi AI, Janardhan V, Hanel RA, Lanzino G (2007) Comparison of endovascular and surgical treatments for intracranial aneurysms: an evidencebased review. Lancet Neurol 6(9):816825

R;

24- R.J. Komotar, J. Mocco, R.A. Solomon Guidelines for the surgical treatment of unruptured intracranial aneurysms: the 
first annual J. Lawrence pool memorial research symposium-controversies in the management of cerebral aneurysms Neurosurgery, 62 (2008), pp. 183-193

25- Rowe A. J., Finlay H. M., Canham P. B. (2003). Collagen biomechanics in cerebral arteries and bifurcations assessed by polarizing microscopy. J. Vasc. Res. 40, 40641510.1159/000072831[PubMed]

26- Ryu C. W., Kwon O. K., Koh J. S., Kim E. J. (2010). Analysis of aneurysm rupture in relation to the geometric indices: aspect ratio, volume, and volume-to-neck

ratio. Neuroradiology.[Epub ahead of $\mathrm{S}$; print]. [PubMed]

27- Shojima M., Oshima M., Takagi K., Torii R., Hayakawa M., Katada K., Morita A., Kirino T. (2004).Magnitude and role of wall shear stress on cerebral aneurysm: computational fluid dynamic study of 20 middle cerebral artery aneurysms. Stroke 35, 2500 250510.1161/01.STR.0000144648.89172

28- Stapf C, Mohr JP, Pile-Spellman J, Sciacca RR, Hartmann A, Schumacher HC, Mast H (2002) Concurrent arterial aneurysms in brain arteriovenous malformations with haemorrhagic presentation. J Neurol Neurosurg Psychiatr 73:294-298

29- Swietaszczyk C., Maciaczyk J., TafilKlawe M., Kasprzak H. A. (2004). what is the origin of cerebral aneurysms? Prz.Lek. 61, 115119 [PubMed]

T;

30- Torner JC, Kassell NF, Wallace RB, Adams HP Jr (1981) Preoperative prognostic factors for rebleeding and survival in aneurysm patients receiving antifibrinolytic therapy: report of the Cooperative Aneurysm Study. Neurosurgery 9:506-513

31-Tulamo, R., J. Frosen, S. Junnikkala, A. Paetau, J. Pitkaniemi, M. Kangasniemi, M. Niemela, J. Jaaskelainen, E. Jokitalo, A. Karatas, J. Hernesniemi, and S. Meri,
Complement activation associates with saccular cerebral arteryaneurysm wall degeneration and rupture.Neurosurgery, 2006. 59(5): p. 1069-76; discussion10767. $\mathrm{V}$;

32- Van der Drift, J. H. A.: Electroencephalography and cerebral tumors. In Magnus, O., et al.: Suppl. No. 19, EEG-Journal, Elsevier, Amsterdam 1961 (pp. 156-159)

W;

33- Wermer MJ, van der Schaaf IC, Algra A, Rinkel GJ (2007) Risk of rupture of unruptured intracranial aneurysms in relation to patient and aneurysm characteristics: an updated meta-analysis. Stroke 38(4):1404-1410. Epub 2007 Mar 1

34- White PM, Wardlaw JM, Teasdale E, Sloss S, Cannon J, Easton V (2001) Power transcranial Doppler ultrasound in the detection of intracranial aneurysms. Stroke 32:1291-1297

35-Wiebers D, Whisnant JP, Huston J III, Meissner I, Brown RD Jr, Piepgras DG, Forbes GS, Thielen K, Nichols D, O'Fallon WM, Peacock J, Jaeger L, Kassell NF, Kongable- Beckman GL, Torner JC (2003) Unruptured intracranial aneurysms: natural history, clinical outcome, and risks of surgical and endovascular treatment. Lancet 2003, 362: p103-110 imaging. J Neurosurg 96:684-689

Y

36-Yaşargil, M.G., Microneurosurgery. Vol I.: Georg Thieme Verlag.In Thompson, R. A., J. R. Green Advances in Neurology. Raven, 1984: Stuttgart (pp. 181-209)

37- Yasuno, K., K. Bilguvar, P. Bijlenga, S.K. Low, B. Krischek, G. Auburger, M. J. Hernesniemi, G.J. Rinkel, H. Zembutsu, I. Inoue, A. Palotie, F. Cambien, Y. Nakamura, R.P. Lifton, and M. Gunel, Genome-wide associationstudy of intracranial aneurysm identifies three new risk loci. Nat Genet, 2010. 42(5): p. 420-5. 
38- Zal. Ghassan Kerry, Alexander Hammer , Anahi Steiner, Gholamreza Ranaie, Ingrid Baer, Christian, Stefan Kunze, Hans-Herbert Steiner Published: Treatment of ruptured intracranial aneurysms yesterday and now, neurosurgery journal,central Europ ,germany,

https://doi.org/10.1371/journal.pone.017 2837 March 3, 2017.
39- zanne M. Dorhout Mees, MD; Andrew J. Molyneux, MD, PhD; Richard S. Kerr, MD, PhD; Ale Algra, MD, PhD; Gabriel J.E. Rinkel, $\mathrm{MD}, \mathrm{PhD}$, University of Oxford and Oxford Radcliffe Hospitals, National Health Service Trust, Radcliffe Infirmary, Oxford, United Kingdom.; Timing of Aneurysm Treatment After Subarachnoid Hemorrhage Relationship With Delayed Cerebral Ischemia and Poor Outcome, Stroke J. 2012;43:21262129.April 4, 2012. 